\title{
Medidor inteligente para las variables de energía eléctrica basado en un sistema embebido
}

\author{
J. Álvarez-Alvarado ${ }^{1}$, G.J. Ríos-Moreno ${ }^{1}$, G. Ronquillo ${ }^{2}$, M. Trejo-Perea ${ }^{1}$ \\ ${ }^{1}$ Universidad Autónoma de Querétaro, Facultad de ingeniería, \\ Santiago de Querétaro, Qro., México \\ ${ }^{2}$ Centro de Ingeniería y Desarrollo Industrial (CIDESI), \\ Santiago de Querétaro, Qro., México
}

jose_manuel_51@hotmail.com, \{riosg,mtp\}@uaq.mx, gronquillo@gmail.com

Resumen. Hoy en día, cualquier sociedad o comunidad que deseen ser sostenible, debe pensar en la conservación y eficiencia de la energía eléctrica, que es un factor ético, donde se requiere el conocimiento del consumo total de energía con el fin de ayudar a preservar el medio ambiente. En este trabajo se presenta un sistema integrado basado en una FPGA para la medición y procesamiento de variables eléctricas y su interfaz para mostrar los datos procesados. Se muestran los resultados del trabajo y la comparación con un instrumento comercial de la marca Fluke 434.

Palabras clave: Sistema embebido, monitor de carga eléctrica, smart metter, FPGA, consumo de energía.

\section{Smart Metter for Electrical Energy Variables Based on an Embedded System}

\begin{abstract}
Nowadays, any society or community that wish to be sustainable, should think about conservation and energy efficiency, which is an ethical factor where require the knowing of the overall energy consumption in order to help preserve the environment. This paper presents an Embedded System based in an FPGA for the measurement and processing of electric variables, and its interface to show the data processed. The results of this system and the comparison with Fluke 434 are shown.
\end{abstract}

Keywords: Embedded system. electric load monitor. smart metter. FPGA. power consumption. 


\section{Introducción}

El conocimiento de la demanda futura de energía eléctrica en una región, en un país o en el mundo, es una herramienta importante para el desarrollo e implementación de una política energética, ya sea por organizaciones internacionales o por el gobierno. Esta demanda de energía tendrá que ser satisfecha por una combinación óptima de las fuentes de energía disponibles, teniendo en cuenta las restricciones impuestas por el futuro cambio económico y social hacia un mundo sostenible [1, 2].

En los últimos años, el tema de la energía ha sido un tema abordado por los investigadores debido a su demanda en rápido crecimiento en todo el mundo. De acuerdo con la International Energy Agency (IEA), el suministro de energía eléctrica podría ser un $50 \%$ mayor en 2030 de lo que hay en la actualidad y esto tendría consecuencias económicas y ambientales alarmantes [3, 4].

Actualmente, el consumo de electricidad en el mundo ha ido en aumento debido a varios factores, que han evolucionado con el tiempo, tanto tecnológico y social. Sin embargo, esto afecta afectando a la sostenibilidad del país, por lo que la necesidad de implementar un sistema de control para reducir este consumo es de gran importancia, ya que pueden alcanzarse no sólo el ahorro económico, sino que le ayudará a cuidar el medio ambiente.

La preocupación creciente entre las naciones industrializadas es el aumento del costo y el consumo de energía eléctrica. Al permitir a los consumidores controlar el consumo de energía de sus dispositivos eléctricos, pueden auxiliar a mejorar la conciencia y la disciplina de los usuarios de los hábitos domésticos [5].

Las computadoras, redes, la medición y el desarrollo de nuevas tecnologías han obtenido un impacto importante en el aspecto de la calidad de la energía. Los avances tecnológicos en redes, comunicaciones, gestión de datos y la tecnología aplicada a la medición de la calidad de energía se han combinado para reducir significativamente el costo de los sistemas de monitoreo y aumentar la capacidad de estos sistemas [6]. El procesamiento y la implementación de hardware de la matriz de puertas de campo programable (FPGA) en paralelo pueden reducir el tiempo total de cálculo para la interpretación de los datos y para ob-ner el estado de la red de distribución [7].

El uso de FPGA en monitoreo de sistemas eléctricos han sido usados en diversas investigaciones, como la propuesta de [8], el cual utiliza el FPGA para la adquisición y de datos para el consumo de energía en cargas individuales, utilizando convertidores análogos-digitales en paralelo, almacenando y enviando los datos a la PC.

El sistema de monitoreo propuesto por [9], realiza la adquisición de datos con un ADC0804, con una resolución de 8bits y almacenando los datos en una FIFO, obteniendo muestras de 60 muestras por ciclo.

El FPGA representa una buena opción para el desarrollo e implementación de monitoreo de energía debido a que permiten el desarrollo rápido de prototipos y para el diseño de complejos sistemas de hardware. Estos dispositivos se han utilizado en muchas aplicaciones reales, Las ventajas de la implementación de este sistema implican un bajo costo, una mayor precisión en la medición de variables eléctricas y un incremento de la operación flexibilidad al proporcionar monitoreo local y remoto a través de Internet [9]. 
La segunda sección de este documento se describe las consideraciones teóricas y los algoritmos propuestos para el cálculo y la programación de las fuentes de energía y parámetros en la FPGA. La tercera sección se encuentran los materiales y métodos aplicados, así como el desarrollo del hardware y software para el sistema propuesto e incluye la validación del sistema implantado y muestra la interfaz de realizada en LabView. La última sección presenta las conclusiones del sistema implementado y el impacto ecológico, social y económico de la investigación.

\section{Consideraciones teóricas}

A medida que la tecnología avanza, se espera que la complejidad de los equipos eléctricos aumenta al hacerlo también con el sistema eléctrico, de hecho, nos encontramos con tres tipos diferentes de cargas en edificios: puramente resistivas, puramente reactivas y parcialmente reactivos. Los instrumentos propuestos utilizan el procesamiento de señal digital para realizar un análisis espectral de la tensión y la corriente.

El método de integración discreta, calcula la corriente y voltajes señales y potencia activa a través de la versión digital de cada fuente de energía, en este orden, respectivamente. También permite el cálculo de los valores eficaces de la tensión, la corriente y, en consecuencia, la potencia aparente y el factor de potencia. Las muestras de corriente y de tensión que se ajustan, uno por uno, a los puntos idénticos en el tiempo, y el tiempo total de muestreo debe ser un número entero de $N$ veces el período fundamental de las señales de entrada.

\subsection{Medición de voltaje y corriente RMS}

RMS se define para señales periódicas a pesar de que se utiliza generalmente para extraer información a partir de mediciones de perturbación sistema de energía que son no periódica. En caso de una transición, el RMS calculadas no da el valor correcto de Voltaje del nuevo estado hasta que la ventana sobre la que el RMS se calcula por completo contiene muestras del nuevo estado.

$$
\begin{gathered}
V R M S=\sqrt{\frac{1}{T} \int_{t_{0}}^{t_{0}+T}(v(t))^{2} d t} \cong \sqrt{\frac{1}{N} \sum_{n-1}^{N-1}(v(t))^{2}}, \\
I R M S=\sqrt{\frac{1}{T} \int_{t_{0}}^{t_{0}+T}(i(t))^{2} d t} \cong \sqrt{\frac{1}{N} \sum_{n-1}^{N-1}(i(t))^{2}} .
\end{gathered}
$$

El valor RMS, que puede ser obtenido con métodos digitales, donde $N$ es el total de las muestras de la señal adquirida digitalmente en observación, y $n$ es un índice de la misma, $v$ e $i$ son los datos adquiridos por los sensores.

El valor RMS de voltaje en su forma discreta tiene las mismas propiedades: el valor eficaz que corresponde a una ventana que contiene las dos muestras de eventos previo 
y posterior dará típicamente un valor de RMS que se encuentra entre la antigua y de la nueva tensión eficaz. Ciertas combinaciones de caída de magnitud y ángulo de fase de desempate incluso conducen a valores eficaces fuera de este rango [10].

\subsection{Validación del sistema}

Para calcular el porcentaje de error de los parámetros eléctricos de corriente, potencia activa tensión, potencia reactiva, potencia aparente y el factor, la siguiente ecuación se define de acuerdo a Cox [11].

$$
\text { \%error }=100\left(\frac{X_{\text {Fluke }}-Y_{\text {system }}}{Y_{\text {system }}}\right),
$$

donde Ysistem indica la medida de los parámetros eléctricos por medio del Sistema desarrollado, Fluke $Y$ representa los parámetros eléctricos medidos por el analizador de calidad de energía 43B.

\section{Implementación del hardware}

En éste proyecto, se propone utilizar un FPGA como una tarjeta de adquisición y procesamiento de datos, debido a que es de arquitectura abierta y a su flexibilidad, permite su reprogramación para diversas aplicaciones. Comparando el dispositivo comercial Fluke, el sistema propuesto es de bajo costo.

\subsection{Características del FPGA}

Para la adquisición y procesamiento de datos, fue seleccionada una FPGA Spartan6 embebida en una tarjeta de Mojo [12]. La tarjeta mojo contiene la Spartan 6 XC6SLX9 FPGA, 84 pines IO digitales, 8 entradas analógicas, 8 focos LED de propósito general, en la regulación de voltaje de la tarjeta puede soportar de $4.8-12 \mathrm{~V}$, un ATmega32U4 utilizado para la configuración de la FPGA, comunicaciones USB, y la lectura de los pines analógicos y memoria flash on-board para almacenar el archivo de configuración de la FPGA.

Es posible realizar pruebas rápidas y obtener actualizaciones de las modificaciones de software individuales con el uso de FPGA y también tienen un coste de producción razonable en relación con su rendimiento.

\subsection{Sensor de voltaje y corriente}

El sensor de corriente utilizada en este proyecto es un núcleo dividido sensor de tipo CT SCT-013-000 (figura 1). El SCT-013 a 030 es un sensor de corriente AC no invasivo que puede detectar un valor de corriente máximo (RMScurrent) de 100A y el secundario del CT da salida a una tensión máxima de $1 \mathrm{~V}$, con 2000 vueltas. Es un sensor salida 
de tensión y por sí mismo viene con una resistencia de carga.[13]. Para determinar la resistencia de carga, se ha calculado el pico principal ( $c c$ ) como 141,4A dado por:

$$
p c=R M S_{\text {current }} * \sqrt{2} \text {. }
$$

El pico de corriente secundaria es de $70,7 \mathrm{~mA}$, obtenido de la siguiente ecuación:

$$
s c=\frac{p c}{N o_{\text {turns }}}
$$

Para ello, la resistencia de carga se calcula mediante:

$$
p c=\frac{2.5}{s c} \text {. }
$$

El principio de funcionamiento de sensor de corriente y el sensor de SCT 013 se muestran en la Fig. 1.

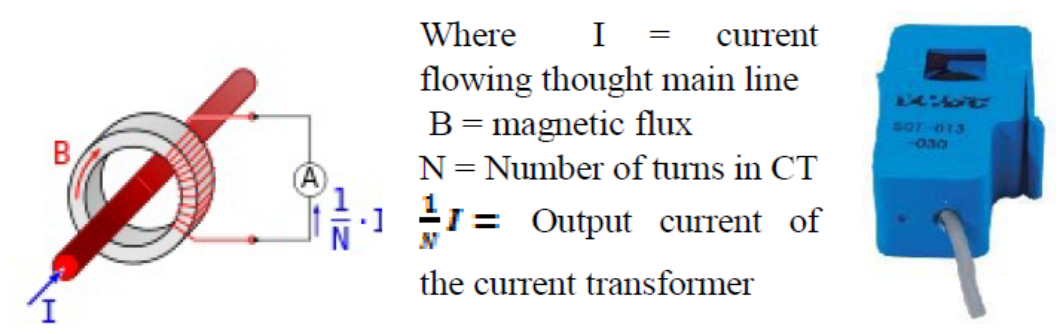

Fig. 1. Concepto básico del transformador de corriente y la imagen real del sensor de corriente (SCT-013-030) [13]

El sensor de tensión es un transformador de tensión, lo que asegura una red de energía protección galvánica. Este transformador se utiliza para convertir una tensión de $120 \mathrm{VAC}$ en una tensión proporcional inferior. Ambas señales de corriente y tensión están montadas a un acondicionador de señal para aumentar el rango dinámico $( \pm 5 \mathrm{~V})$, para luego ser enviadas a un filtro pasa-bajas antes de completar la conversión de analógico a digital (A / D).

\subsection{Interfaz digital}

Para este proyecto, se utilizó el software LabVIEW, para hacer el análisis de las señales. Fig 2 muestra el circuito de acondicionamiento para montar la señal de Voltaje al ADS, se utiliza un amplificador operacional (LM358AD) para montar la señal de tensión en el rango positivo.

El sensor de corriente se conecta a un cable aux y en paralelo con el sensor de carga, para obtener la lectura de la señal en la interfaz de LabView.. 


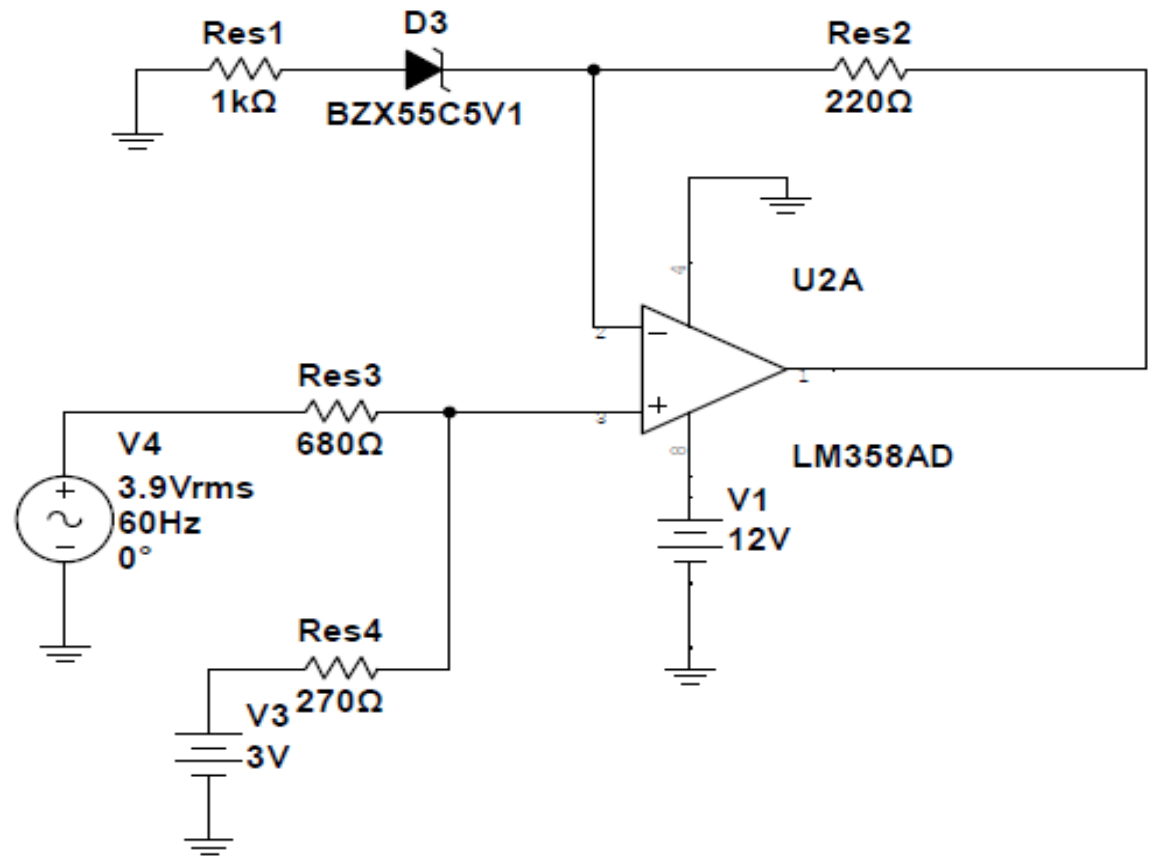

Fig. 2. Circuito de acondicionamiento de voltaje

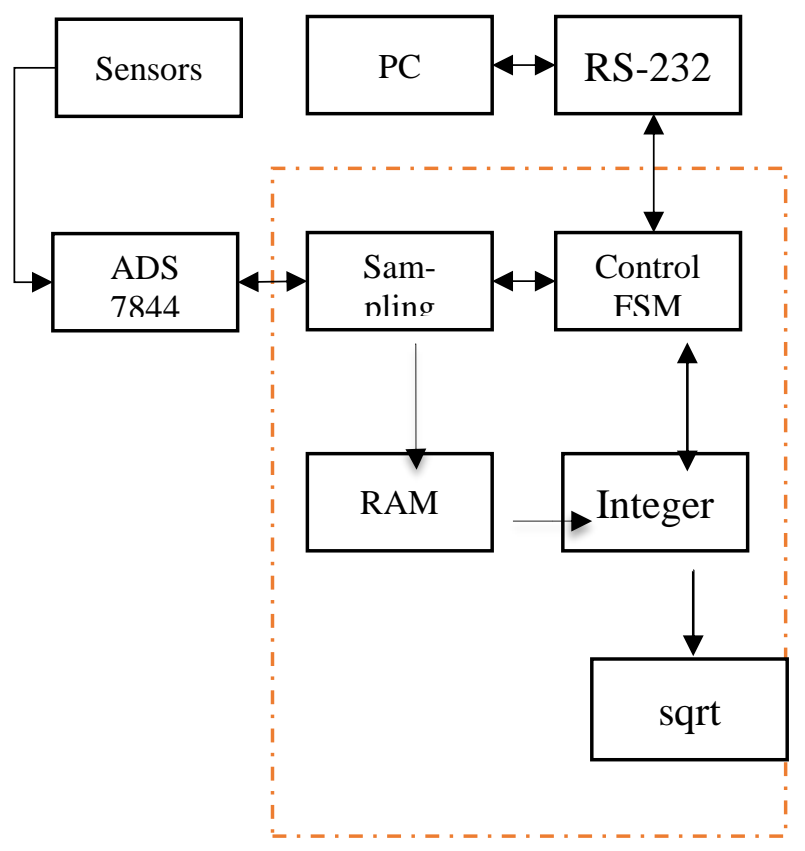

Fig. 3. Descripción general de la arquitectura 


\subsection{Implementación en hardware}

Las señales de tensión y corriente pasan a través de un filtro antialiasing pasa-bajas analógico antes de completar la conversión de analógico a digital. Un DAQ realiza las conversiones entre las señales analógicas en señales digitales; un diagrama de bloques detallado de la DAQ se muestra en la Fig. 3. El convertidor analógico-digital seleccionado es un ADS7844 de 8 canales, 12 bits de resolución con una disipación de potencia de $3 \mathrm{~mW}$ a una velocidad máxima de muestreo de $200 \mathrm{kHz}$ y una alimentación de hasta $+5 \mathrm{~V}$. El dispositivo que se utiliza para generar las señales de control ADC, establece la frecuencia de muestreo, almacena los datos, y la interfaz con el PC es el mojo FPGA Spartan-6. Como se muestra en la Fig. 3, los bloques funcionales se implementaron en el FPGA, basados en el trabajo de [9] y se explican posteriormente.

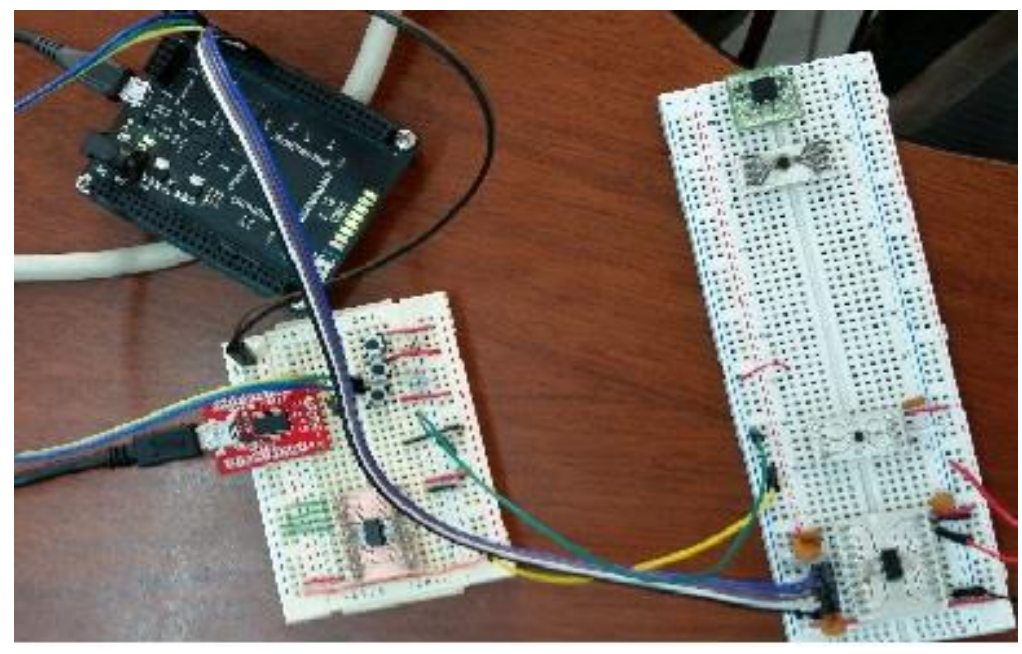

Fig. 4. Circuito de voltaje

- Control de la FSM: Este módulo es una máquina de estados finitos que genera las señales digitales requeridas por el ADC para iniciar un nuevo ciclo de conversión y almacenar los valores resultantes de estas conversiones en la memoria RAM, implementada en el FPGA.

- RAM: Cada valor convertido se almacena en el buffer de memoria y espera ser transmitido al equipo host a través del controlador periférico USB. Este módulo es una memoria RAM implementada y controlada por el FPGA.

- Base de tiempo: Este módulo establece la velocidad de muestreo de la conversión de analógico a digital. La frecuencia de muestreo se fijó en 1,2 kHz.

\subsection{Simulaciones}

En la Fig. 4. Muestra un circuito final, que muestra la tarjeta mojo, el circuito de acondicionamiento y el ADC para la adquisición de datos. Para hacer las medidas, era 
necesario conocer el factor de potencia del circuito eléctrico. El banco de prueba se seleccionó una carga resistiva, para controlar la corriente y el voltaje.

La Fig. 5 muestra la adquisición de datos de tensión y la corriente eléctrica del sistema basado en FPGA y el analizador de calidad de energía eléctrica Fluke434 en una línea de tensión eléctrica en un banco de pruebas. Se realiza una comparación mostrando los valores reales del Fluke434 y los datos adquiridos en la interfaz gráfica por el sistema basado en FPGA que puede mostrar RMS de tensión y corriente eléctrica. El Fluke fue seleccionado para realizar la comparación entre los resultados obtenidos del mismo contra el sistema propuesto basado en FPGA, debido a que se ha utilizado en diversos trabajos como [9], [14].

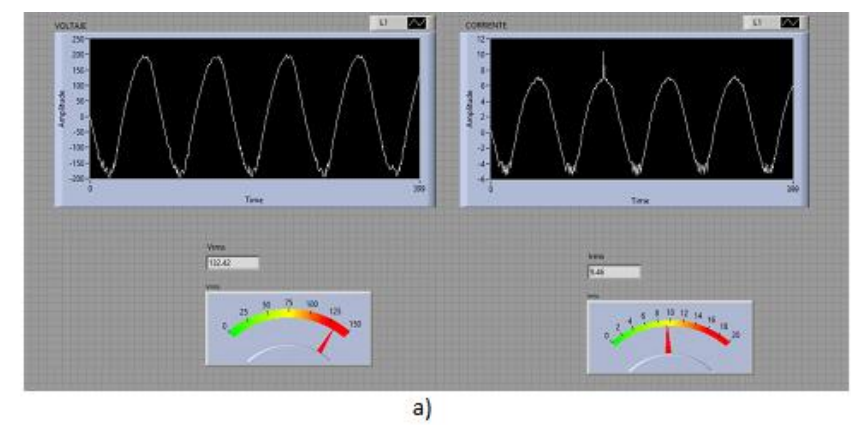

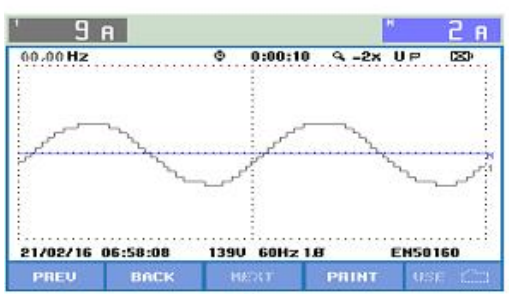

b)

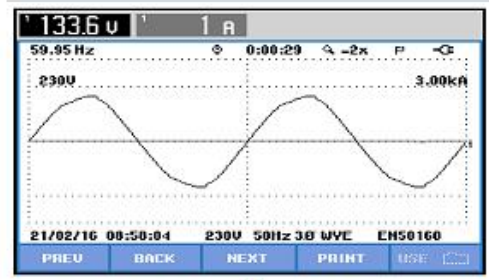

Fig. 5. Las mediciones de corriente y voltaje del sistema se muestran en a). En b) y c) se encuentran de las mediciones obtenidas con el analizador de calidad comercial Fluke para las variables de alimentación de corriente y tensión respectivamente

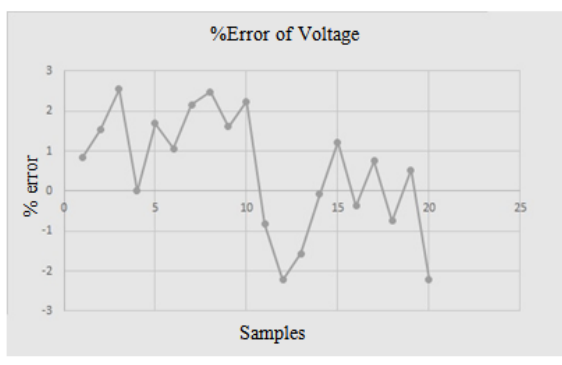

a)

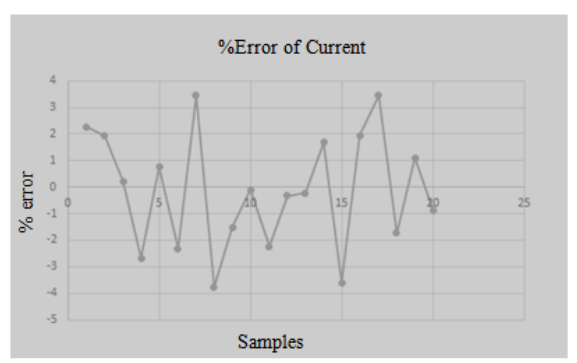

Fig. 6. Error measurements for voltage a) and current b) 
Para la comparación y cálculo del error de la Ec. 3, se seleccionaron 20 mediciones basándose en [15] de acuerdo con el fin de ver los errores de tensión y corriente, y luego aplicar la ecuación (3). La Fig. 6 muestra el porcentaje de error del sistema.

\section{Conclusiones y resultados}

La principal característica del sistema de control propuesta es la flexibilidad y bajo costo. La flexibilidad garantiza que el software y el hardware puedan ser modificados y adaptados a diferentes mediciones o aplicaciones futuras, incluso para realizar estrategias de control. Así mismo, este sistema es comparativamente más fácil de calibrar y su reproducción digital es de mayor resolución, no causa problemas de linealidad que podría ocurrir en los contadores de energía basados en la reproducción analógica. El uso de sensores no invasivos permite la protección del sistema y para el usuario, si modificar la arquitectura eléctrica.

El ahorro sistema de monitoreo de energía eléctrica muestra una tasa de error por debajo de $3 \%$ y $4 \%$ de acuerdo a la Ec. 3 para las señales de tensión y corriente, respectivamente, que depende de la precisión de los convertidores analógicos digitales y el acondicionamiento de la señal analógica. Las fluctuaciones observadas pueden ser debidas a que se tomaron los valores comerciales, y no reales de la etapa de acondicionamiento de la señal.

Este sistema tiene como objetivo ser parte del diseño de un tablero eléctrico inteligente, con el fin de tener ahorros potenciales en la energía y económico, con la adición de un sistema experto como estrategia de control.

El sistema basado en FPGA propuesto resulta más económico y de una arquitectura abierta, por lo cual, podremos añadir diversas funciones al sistema propuesto en futuros trabajos de investigación.

Agradecimientos. Agradecemos especialmente al CONACYT (Consejo Nacional de Ciencia y Tecnología) por el apoyo económico y técnico, y a la Facultad de Ingeniería de la Universidad Autónoma de Querétaro.

\section{Referencias}

1. Morales-Acevedo, A.: ISES Solar World Congress Forecasting future energy demand: Electrical energy in Mexico as an example case. Energy Procedia. Vol. 57, pp. 782-790 (2014)

2. Álvarez-Alvarado, J., Trejo-perea, M., Herrera-Arellano, M., Ríos-moreno, J.G.: Control Strategies of Electrical Power on Smart Buildings , a Review. Int. Rev. Electr. Eng. Vol. 10, pp. 764-770 (2015)

3. Moslehi, K., Kumar, R.: Smart grid - A reliability perspective. In: Innov. Smart Grid Technol. Conf. ISGT, pp. 1-8 (2010)

4. Iea, I.E.A.: World Energy Outlook, http://link.aip.org/link/ELPWAQ/v23/i4/p329/ s3\&Agg=doi. 
5. Marchman, C., Bertels, J., Gibbs, D., Students, S.N., Advisor, K.K.: Remote Monitoring and Control of Residential and Commercial Energy Use. In: Int. Telemetering Conf. Proc. pp. 1-8 (2014)

6. Trejo-Perea, M., Herrera-Ruiz, G., Rios-Moreno, J., Miranda, R.C., Araiza, E.R.: Greenhouse Energy Consumption Prediction using Neural Networks Models. Int. J. Agric. Biol. Vol. 11, pp. 1-6 (2009)

7. Depuru, S.S.S.R., Wang, L., Devabhaktuni, V.: Smart meters for power grid: Challenges, issues, advantages and status. Renew. Sustain. Energy Rev. Vol. 15, pp. 2736-2742 (2011)

8. Remscrim, Z., Paris, J., Leeb, S.B., Shaw, S.R., Neuman, S., Schantz, C., Muller, S., Page, S.: FPGA-based spectral envelope preprocessor for power monitoring and control. In: Conf. Proc. IEEE Appl. Power Electron. Conf. Expo. APEC, pp. 2194-2201 (2010)

9. Trejo-Perea, M., Herrera-Ruiz, G., Vargas-Vázquez, D., Luna-Rubio, R., Rios-Moreno, G.J.: System Electrical Power Monitoring Manifold Based on Software Development and an Embedded System for Intelligent Buildings. J. Energy, Vol. 137, pp. 1-10 (2011)

10. Styvaktakis, E.: Automatic classification of power system events using RMS voltage measurements. In: Power Eng. Soc. Summer Meet, pp. 824-829 (2002)

11. Cox, M.D., Williams, T.B.: Induction Varhour and Solid-State Varhour Meters Performances On Nonlinear Loads, Vol. 5, pp. 1678-1686 (1990)

12. Micro, E.: Mojo SPARTAN-6, https://embeddedmicro.com/tutorials/mojo.

13. Shajahan, A.H., Anand, A.: Data acquisition and control using Arduino-Android platform: Smart plug. Int. Conf. Energy Effic. Technol. Sustain. ICEETS, pp. 241-244 (2013)

14. Gonzalez-Gutierrez, C.A., Rodriguez-Resendiz, J., Mota-Valtierra, G., Rivas-Araiza, E.A., Mendiola-Santibañez, J.D., Luna-Rubio, R.: A PC-based architecture for parameter analysis of vector-controlled induction motor drive. Comput. Electr. Eng., Vol. 37, pp. 858-868 (2011)

15. Trejo Perea, M.: Monitoreo y ahorro de energía para edificios inteligentes. (2008) 